\title{
A NONCOMMUTATIVE DE FINETTI THEOREM: INVARIANCE UNDER QUANTUM PERMUTATIONS IS EQUIVALENT TO FREENESS WITH AMALGAMATION
}

\author{
CLAUS KÖSTLER AND ROLAND SPEICHER ${ }^{(\dagger)}$
}

\begin{abstract}
We show that the classical de Finetti theorem has a canonical noncommutative counterpart if we strengthen "exchangeability" (i.e., invariance of the joint distribution of the random variables under the action of the permutation group) to invariance under the action of the quantum permutation group. More precisely, for an infinite sequence of noncommutative random variables $\left(x_{i}\right)_{i \in \mathbb{N}}$, we prove that invariance of the joint distribution of the $x_{i}$ 's under quantum permutations is equivalent to the fact that the $x_{i}$ 's are identically distributed and free with respect to the conditional expectation onto the tail algebra of the $x_{i}$ 's.
\end{abstract}

\section{INTRODUCTION}

The de Finetti theorem states that an infinite family of random variables whose distribution is invariant under finite permutations (such a family is called exchangeable) is independent and identically distributed with respect to the conditional expectation onto the tail algebra of the random variables. Since the implication in the other direction is fairly elementary one has the equivalence between exchangeability and conditional independence. See, e.g., Kal for an exposition on the classical de Finetti theorem.

In a noncommutative context classical random variables are replaced by, typically noncommuting, operators on Hilbert spaces. The expectation with respect to a probability measure is then replaced by a state on the algebra generated by these operators. The notion of exchangeability makes of course also sense in such a context, as invariance of mixed moments under permutations of the random variables, and one can ask what exchangeability implies in such a more general context. It turns out that in the noncommutative world there are actually many quite different possibilities for exchangeable random variables. It was shown in [Koe1 that they all possess some kind of factorization property; but, as one sees from the variety of examples, one cannot expect that exchangeability implies some fixed kind of independence. Indeed, both independence and freeness provide basic examples for exchangeable random variables. (See also Leh, Koe2] for more on this.)

Date: July 3, 2008.

2000 Mathematics Subject Classification. 46L54 (46L65, 46L53, 60G09).

Key words and phrases. Free probability, quantum exchangeability, quantum permutation group.

$\dagger$ Research supported by Discovery and LSI grants from NSERC (Canada) and by a Killam Fellowship from the Canada Council for the Arts. 
However, if one moves into the noncommutative realm, one should also take into account that invariance under permutations is a commutative concept and should be replaced by its noncommutative analogue. To provide such noncommutative analogues of actions of groups was one of the motivations for the creation of the theory of quantum groups, which has been developed very extensively within the last 20 years or so. In particular, Wang introduced in Wan the noncommutative analogue of the permutation group $S_{n}$, namely the quantum permutation group $A_{s}(n)$. So if one considers noncommuting random variables, it is natural to replace the requirement of invariance under permutations by the stronger requirement of invariance under quantum permutations. Classical (commuting) independent random variables do not satisfy this stronger form of exchangeability any more and, as we will show in our main theorem, this noncommutative version of exchangeability singles out again a very special situation - namely freeness with amalgamation. In the same way as classical exchangeability is equivalent to conditional independence, quantum exchangeability is equivalent to freeness with amalgamation.

Thus our noncommutative de Finetti theorem is another instance of the general philosophy that freeness plays in the noncommutative world the same role as independence plays in the commutative world. Note that freeness is not a hidden assumption in our de Finetti theorem, but it is a consequence of replacing the commutative permutation group by its noncommutative counterpart.

Here is the statement of our noncommutative de Finetti theorem. All relevant notions will be defined in Sections 2 and 4 .

Theorem 1.1. Let $(\mathcal{A}, \varphi)$ be a $W^{*}$-probability space and consider an infinite sequence $\left(x_{i}\right)_{i \in \mathbb{N}}$ in $\mathcal{A}$. Then the following two statements are equivalent:

(a) The joint distribution of $\left(x_{i}\right)_{i \in \mathbb{N}}$ with respect to $\varphi$ is invariant under quantum permutations.

(b) The sequence $\left(x_{i}\right)_{i \in \mathbb{N}}$ is identically distributed and free with respect to the conditional expectation $E$ onto the tail algebra of the $\left(x_{i}\right)_{i \in \mathbb{N}}$.

To be precise, $E$ denotes the $\varphi_{\infty}$-preserving conditional expectation from $\mathcal{A}_{\infty}$ onto the tail algebra $\mathcal{A}_{\text {tail }}$ of $\left(x_{i}\right)_{i \in \mathbb{N}}$, where $\mathcal{A}_{\infty}$ is the von Neumann subalgebra generated by $\left(x_{i}\right)_{i \in \mathbb{N}}$ and $\varphi_{\infty}$ is the restriction of $\varphi$ to $\mathcal{A}_{\infty}$. We want to point out that, if $\varphi$ is a trace, then $E$ can be chosen to be the $\varphi$-preserving conditional expectation from $\mathcal{A}$ onto $\mathcal{A}_{\text {tail }}$. More care is needed in the general case of a nontracial state. Here we need to ensure the existence of the conditional expectation $E$. As we will show in Section 4, this can always be achieved for exchangeable random variables, after restriction to the $\mathrm{W}^{*}$-probability space $\left(\mathcal{A}_{\infty}, \varphi_{\infty}\right)$.

Our paper is organized as follows. In the next section we collect the preliminaries. On one side, we present the definition of the quantum permutation group and the notion of invariance under quantum permutations. On the other side, we recall the basic definitions and relevant results about free independence with amalgamation. In Section 3, we will prove the "easy" implication of our de Finetti theorem, namely that freeness with amalgamation implies invariance under quantum permutations. This is actually not as elementary as in the classical case (where it follows directly from the fact that independence is a rule for expressing mixed moments in terms of moments of the single random variables) and we will have to use some of the basic theory of freeness for this proof. In Section 4, we will define the tail algebra of our sequence of random variables, and show some basic properties of the corresponding conditional expectation. Section 5 will finally give the proof of the other implication 
of our de Finetti theorem, Theorem 1.1. The paper closes with an example which shows that, as in the classical case, one needs infinitely many random variables in our de Finetti theorem: quantum exchangeability of finitely many random variables does not necessarily imply freeness with amalgamation.

\section{Preliminaries}

2.1. Noncommutative probability spaces and distributions of random variables. Here we recall the basic notions of non-commutative probability spaces and distributions of random variables; this is just to have a convenient language for our main statements.

Definition 2.1. 1) A noncommutative probability space $(\mathcal{A}, \varphi)$ consists of a unital algebra $\mathcal{A}$ and a unital linear functional $\varphi$.

2) A $W^{*}$-probability space $(\mathcal{A}, \varphi)$ is a von Neumann algebra $\mathcal{A}$ together with a faithful normal state $\varphi$ on $\mathcal{A}$.

Note that for a $W^{*}$-probability space we do not require that our state $\varphi$ is a trace.

Definition 2.2. Let $(\mathcal{A}, \varphi)$ be a non-commutative probability space and $\left(x_{i}\right)_{\in \in \mathbb{N}}$ a sequence in $\mathcal{A}$. The joint distribution of $\left(x_{i}\right)_{i \in \mathbb{N}}$ is given by the collection of all moments $\varphi\left(x_{i(1)} \cdots x_{i(n)}\right)$ for all $n \in \mathbb{N}$ and all $i(1), \ldots, i(n) \in \mathbb{N}$.

2.2. Quantum Permutation Group. Wang introduced in Wan the following noncommutative version of the permutation group $S_{n}$.

Definition 2.3. The quantum permutation group $A_{s}(n)$ is defined as the universal unital $C^{*}$-algebra generated by elements $u_{i j}(i, j=1, \ldots, n)$ such that we have

- each $u_{i j}$ is an orthogonal projection: $u_{i j}^{*}=u_{i j}=u_{i j}^{2}$ for all $i, j=1, \ldots, n$

- the elements in each row and column of $u=\left(u_{i j}\right)_{i, j=1}^{n}$ form a partition of unity, i.e., are orthogonal and sum up to 1 : for each $i=1, \ldots, n$ and $k \neq l$ we have

$$
u_{i k} u_{i l}=0 \quad \text { and } \quad u_{k i} u_{l i}=0 ;
$$

and for each $i=1, \ldots, n$ we have

$$
\sum_{k=1}^{n} u_{i k}=1=\sum_{k=1}^{n} u_{k i}
$$

Note that the above requirements imply in particular that the matrix $u=$ $\left(u_{i j}\right)_{i, j=1}^{n}$ is orthogonal, i.e., for each $i, j=1, \ldots, n$ we have

$$
\sum_{k=1}^{n} u_{i k} u_{j k}=\delta_{i j} 1 \quad \text { and } \quad \sum_{k=1}^{n} u_{k i} u_{k j}=\delta_{i j} 1 .
$$

$A_{s}(n)$ is a compact quantum group in the sense of Woronowicz Wor. That this is the right noncommutative version of the permutation group can be seen from the fact that adding commutativity of the $u_{i j}$ to the above definition yields the group algebra of the permutation group and that, by a theorem of Wang [Wan, $A_{s}(n)$ is the biggest Hopf algebra coacting on a space of $n$ points. For more information on $A_{s}(n)$, see $\mathrm{BC}, \mathrm{BBC}$.

For $n=1,2,3$ the quantum permutation group is the same as the usual permutation group, i.e., in these cases $A_{s}(n)$ is isomorphic as a Hopf algebra to $\mathbb{C} S_{n}$. 
For $n \geq 4$, however, the quantum version is strictly larger than the classical one; this can be seen, for example, by finding representations of the $u_{i j}$ which do not commute. Here is such a representation in the case $n=4$ :

$$
u=\left(\begin{array}{cccc}
q_{1} & 1-q_{1} & 0 & 0 \\
1-q_{1} & q_{1} & 0 & 0 \\
0 & 0 & q_{2} & 1-q_{2} \\
0 & 0 & 1-q_{2} & q_{2}
\end{array}\right),
$$

where $q_{1}$ and $q_{2}$ are arbitrary projections. If we take them non-commuting, then the $C^{*}$-algebra generated by $q_{1}$ and $q_{2}$, which is a quotient of $A_{s}(4)$, is infinite dimensional.

Definition 2.4. Consider a noncommutative probability space $(\mathcal{A}, \varphi)$ and a sequence of random variables $\left(x_{i}\right)_{i \in \mathbb{N}}$ in $\mathcal{A}$. We say that the joint distribution (with respect to $\varphi$ ) of this sequence is invariant under quantum permutations or that the sequence is quantum exchangeable if, for any $k \in \mathbb{N}$, the natural action of $A_{s}(k)$ on the $k$-tuple $\left(x_{1}, \ldots, x_{k}\right)$, given by

$$
x_{i} \mapsto \tilde{x}_{i}:=\sum_{j=1}^{k} u_{i j} \otimes x_{j} \in A_{s}(k) \otimes \mathcal{A},
$$

does not change the distribution, i.e., the joint distribution of the $k$-tuple $\left(x_{1}, \ldots, x_{k}\right)$ with respect to $\varphi$ is the same as the joint distribution of the $k$-tuple $\left(\tilde{x}_{1}, \ldots, \tilde{x}_{k}\right)$ with respect to id $\otimes \varphi$.

More explicitly, this means: for all $k, n \in \mathbb{N}$ and all $1 \leq i(1), \ldots, i(n) \leq k$ we have

$$
\varphi\left(x_{i(1)} \cdots x_{i(n)}\right)=\sum_{j(1), \ldots, j(n)=1}^{k} u_{i(1) j(1)} \cdots u_{i(n) j(n)} \cdot \varphi\left(x_{j(1)} \cdots x_{j(n)}\right)
$$

as an equality in $A_{s}(k)$.

To say it in other words, invariance under quantum permutations asks for the validity of (11) for any matrix $u=\left(u_{i, j}\right)_{i, j=1}^{k}$ whose entries are bounded operators on some Hilbert space and satisfy the defining relations of $A_{s}(k)$ from Definition 2.3. Note that we do not apply a state on the elements from $A_{s}(k)$ to get equality in (11), but ask for an algebraic identity in $A_{s}(k)$.

For a permutation $\sigma \in S_{k}$ the permutation matrix $\left(e_{i j}\right)_{i, j=1}^{k}$ with $e_{i j}=\delta_{\sigma(i) j}$ provides an example of such a $u$, in this case (1) gives

$$
\begin{aligned}
\varphi\left(x_{i(1)} \cdots x_{i(n)}\right) & =\sum_{j(1), \ldots, j(n)=1}^{k} \delta_{\sigma(i(1)) j(1)} \cdots \delta_{\sigma(i(n)) j(n)} \varphi\left(x_{j(1)} \cdots x_{j(n)}\right) \\
& =\varphi\left(x_{\sigma(i(1))} \cdots x_{\sigma(i(n))}\right)
\end{aligned}
$$

which is just the invariance of the distribution of $\left(x_{i}\right)_{i \in \mathbb{N}}$ under the permutation $\sigma$. Thus invariance under quantum permutations includes in particular invariance under permutations; quantum exchangeable random variables are in particular exchangeable. 
2.3. Freeness with Amalgamation. Here we collect the basic definitions and needed facts about freeness. For general introductions on free probability theory, see $\mathrm{VDN}, \mathrm{NS}, \mathrm{HP}$. In the classical de Finetti theorem we do not get ordinary independence of the random variables, but have to condition this over the tail algebra. In the same spirit, in our noncommutative de Finetti theorem, we cannot hope for ordinary freeness with respect to the state $\varphi$, but must expect that we have to condition this with respect to the tail algebra of the random variables. Voiculescu introduced such a conditional version of freeness (called operator-valued freeness or freeness with amalgamation) from the very beginning and developed its basic theory in Voi]. In Spe this concept was treated from the combinatorial point of view and it was shown that the theory of free cumulants extends to the operator-valued frame. As our proof of the "easy" direction of theorem (1.1) relies on free cumulants, we will below recall the relevant facts about operator-valued free cumulants.

Let us first give the definition of an operator-valued probability space and freeness. This will be done in a general, algebraic context, as one implication of our de Finetti theorem does only require such general structure.

Recall that a conditional expectation $E: \mathcal{A} \rightarrow \mathcal{B}$ (for unital algebras $\mathcal{B} \subset \mathcal{A}$ ) is a linear map which satisfies $E[b]=b$ for all $b \in \mathcal{B}$ and the bimodule property

$$
E\left[b_{1} a b_{2}\right]=b_{1} E[a] b_{2} \quad \text { for all } b_{1}, b_{2} \in \mathcal{B} \text { and for all } a \in \mathcal{A} \text {. }
$$

Definition 2.5. 1) An operator-valued probability space $(\mathcal{A}, E: \mathcal{A} \rightarrow \mathcal{B})$ consists of a unital algebra $\mathcal{A}$, a unital subalgebra $\mathcal{B} \subset \mathcal{A}$ and a conditional expectation $E: \mathcal{A} \rightarrow \mathcal{B}$. Elements in $\mathcal{A}$ are called (operator-valued) random variables.

2) For a unital algebra $\mathcal{B}$ we denote by $\mathcal{B}\langle X\rangle$ the $\mathcal{B}$-valued polynomials in the formal variable $X$; these are linear combinations of elements of the form $b_{0} X b_{1} X \cdots b_{n-1} X b_{n}$ for all $n=0,1,2, \ldots$ and all $b_{0}, \ldots, b_{n} \in \mathcal{B}$. (For $n=0$, this is just $b_{0}$.) Elements from $\mathcal{B}$ do not commute with $X$ (with the exception of $1 \cdot X=X=X \cdot 1$ ). For $p \in \mathcal{B}\langle X\rangle$ and $a \in \mathcal{A}$ (for some algebra $\mathcal{A}$ which contains $\mathcal{B}$ as a subalgebra) we denote by $p(a) \in \mathcal{A}$ the element which one gets by replacing the variable $X$ by $a$.

3) Let $(\mathcal{A}, E: \mathcal{A} \rightarrow \mathcal{B})$ be an operator-valued probability space and $\left(x_{i}\right)_{i \in \mathbb{N}}$ a sequence of random variables in $\mathcal{A}$. We say that the sequence is identically distributed (with respect to $E$ ) if for each $p \in \mathcal{B}\langle X\rangle$ the expression $E\left[p\left(x_{i}\right)\right]$ does not depend on $i \in \mathbb{N}$.

In the case of an ordinary noncommutative probability space, i.e., $\mathcal{B}=\mathbb{C}$ and $E=\varphi$, the $b_{i}$ in the definition of $\mathcal{B}\langle X\rangle=\mathbb{C}\langle X\rangle$ are superfluous and $\mathbb{C}\langle X\rangle$ are just ordinary polynomials; in this case "identically distributed" just means that for each $n \in \mathbb{N}$ the ordinary moment $\varphi\left(x_{i}^{n}\right)$ does not depend on $i$.

Definition 2.6. Let $(\mathcal{A}, E: \mathcal{A} \rightarrow \mathcal{B})$ be an operator-valued probability space and $I$ an arbitrary index set. Random variables $\left(a_{i}\right)_{i \in I}$ are called free with respect to $E$ (or free with amalgamation over $\mathcal{B}$ ) if we have for all $n \in \mathbb{N}$, all $i(1), \ldots, i(n) \in I$ with $i(1) \neq i(2) \neq \cdots \neq i(n)$ and all $\mathcal{B}$-valued polynomials $p_{1}, \ldots, p_{n} \in \mathcal{B}\langle X\rangle$ with $E\left[p_{m}\left(a_{i(m)}\right)\right]=0(m=1, \ldots, n)$ that also

$$
E\left[p_{1}\left(a_{i(1)}\right) \cdots p_{n}\left(a_{i(n)}\right)\right]=0 .
$$

The special case where $\mathcal{B}$ is $\mathbb{C}$ (and thus $E$ a unital linear functional $\varphi: \mathcal{A} \rightarrow \mathbb{C}$ ) gives just the usual definition of freeness. 
2.4. Operator-valued free cumulants. The combinatorial theory of operatorvalued freeness Spe relies on the notions of non-crossing partitions and free cumulants. We will now recall these notions.

Definition 2.7. 1) A partition $\pi$ of a set $S$ is a decomposition $\pi=\left\{V_{1}, \ldots, V_{r}\right\}$ of $S$ into disjoint, non-empty subsets $V_{i}$. The elements $V_{i}$ are called the blocks of $\pi$. We denote the partitions of $S$ by $\mathcal{P}(S)$. In the case $S=\{1, \ldots, n\}$, we just write $\mathcal{P}(n)$.

2) For $\pi, \sigma \in \mathcal{P}(n)$ we say that $\pi \leq \sigma$ if each block of $\pi$ is contained in a block of $\sigma$.

2) Let $S$ be an ordered set. A partition $\pi \in \mathcal{P}(S)$ is called non-crossing if there do not exist two different blocks $V, W$ of $\pi$ such that we have $s_{1}<t_{1}<s_{2}<t_{2}$ and $s_{1}, s_{2} \in V$ and $t_{1}, t_{2} \in W$. The set of non-crossing partitions of $S$ is denoted by $N C(S)$, or just $N C(n)$ in the case of $S=\{1, \ldots, n\}$.

If one draws partitions by connecting elements belonging to the same block by half-circles below the numbers $1, \ldots, n$, then the partition is non-crossing if and only if one does not get crossings between different blocks in such a drawing. Another characterization of a non-crossing partition is the following recursive description: $\pi \in \mathcal{P}(S)$ is non-crossing if at least one of the blocks of $\pi$, say $V$, is an interval (i.e., consists of consecutive numbers) and if $\pi \backslash V$ is a non-crossing partition of $S \backslash V$.

Definition 2.8. Let $(\mathcal{A}, E: \mathcal{A} \rightarrow \mathcal{B})$ be an operator-valued probability space.

1) A map $\rho: \mathcal{A}^{n} \rightarrow \mathcal{B}$ (for $n \in \mathbb{N}$ ) is called a $\mathcal{B}$-functional if it is $n$-linear and if we have for all $b_{0}, \ldots, b_{n} \in \mathcal{B}$ and all $a_{1}, \ldots, a_{n} \in \mathcal{A}$ that

$$
\rho\left(b_{0} a_{1} b_{1}, a_{2} b_{2}, \ldots, a_{n-1} b_{n-1}, a_{n} b_{n}\right)=b_{0} \rho\left(a_{1}, b_{1} a_{2}, \ldots, b_{n-2} a_{n-1}, b_{n-1} a_{n}\right) b_{n} .
$$

2) Let, for each $k \in \mathbb{N}$, a $\mathcal{B}$-functional $\rho_{k}: \mathcal{A}^{k} \rightarrow \mathcal{B}$ be given. Then, for $n \in \mathbb{N}$ and $\pi \in N C(n)$ we define a $\mathcal{B}$-functional $\rho_{\pi}: \mathcal{A}^{n} \rightarrow \mathcal{B}$ recursively as follows. If $\pi$ is the maximal element $1_{n} \in N C(n)$, which has only one block, then we put for all $a_{1}, \ldots, a_{n} \in \mathcal{A}$

$$
\rho_{1_{n}}\left[a_{1}, \ldots, a_{n}\right]=\rho_{n}\left(a_{1}, \ldots, a_{n}\right) .
$$

Otherwise, let $V=(i+1, \ldots, i+r)$ be an interval of $\pi$. Then, for $a_{1}, \ldots, a_{n} \in \mathcal{A}$,

$$
\rho_{\pi}\left[a_{1}, \ldots, a_{n}\right]=\rho_{\pi \backslash V}\left[a_{1}, \ldots, a_{i-1}, a_{i} \cdot \rho_{r}\left(a_{i+1}, \ldots, a_{i+r}\right), a_{i+r+1}, \ldots, a_{n}\right]
$$

As illustration of this definition consider

$$
\pi=\{\{1,10\},\{2,5,9\},\{3,4\},\{6\},\{7,8\}\} \in N C(10),
$$

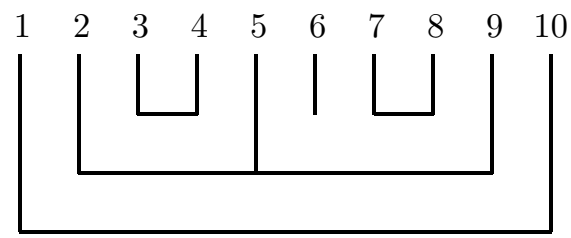

The corresponding $\rho_{\pi}$ is

$$
\rho_{\pi}\left[a_{1}, \ldots, a_{10}\right]=\rho_{2}\left(a_{1} \cdot \rho_{3}\left(a_{2} \cdot \rho_{2}\left(a_{3}, a_{4}\right), a_{5} \cdot \rho_{1}\left(a_{6}\right) \cdot \rho_{2}\left(a_{7}, a_{8}\right), a_{9}\right), a_{10}\right) .
$$


Definition 2.9. Let $(\mathcal{A}, E: \mathcal{A} \rightarrow \mathcal{B})$ be an operator-valued probability space. The corresponding operator-valued free cumulants $\left(\kappa_{n}^{E}\right)_{n \in \mathbb{N}}$ are defined recursively by the moment-cumulant formulas: for each $n \in \mathbb{N}$ and all $a_{1}, \ldots, a_{n} \in \mathcal{A}$ we have

$$
E\left[a_{1} \cdots a_{n}\right]=\sum_{\pi \in N C(n)} \kappa_{\pi}^{E}\left[a_{1}, \ldots, a_{n}\right] .
$$

Note that in the moment-cumulant formula (2) the right hand side is of the form $\kappa_{n}^{E}\left(a_{1}, \ldots, a_{n}\right)$ plus products of lower order terms; thus this can indeed recursively be solved for the $\kappa_{n}^{E}$. There is a quite a lot one can say about the structure of the formulas for the $\kappa_{n}^{E}$, but we will not need this here and refer for more information on this to [NS, Spe. Here are as examples just the first three cumulants:

$$
\kappa_{1}^{E}\left(a_{1}\right)=E\left[a_{1}\right], \quad \kappa_{2}^{E}\left(a_{1}, a_{2}\right)=E\left[a_{1} a_{2}\right]-E\left[a_{1}\right] \cdot E\left[a_{2}\right]
$$

and

$$
\begin{aligned}
\kappa_{3}^{E}\left(a_{1}, a_{2}, a_{3}\right)=E\left[a_{1} a_{2} a_{3}\right]-E\left[a_{1}\right] \cdot & E\left[a_{2} a_{3}\right]-E\left[a_{1} \cdot E\left[a_{2}\right] \cdot a_{3}\right] \\
& -E\left[a_{1} a_{2}\right] \cdot E\left[a_{3}\right]+2 E\left[a_{1}\right] \cdot E\left[a_{2}\right] \cdot E\left[a_{3}\right] .
\end{aligned}
$$

The main result which we will use about free cumulants is that they characterize freeness via the property "vanishing of mixed cumulants".

Theorem 2.10 (阿). Let $(\mathcal{A}, E: \mathcal{A} \rightarrow \mathcal{B})$ be an operator-valued probability space and consider, for some index set $I$, random variables $\left(a_{i}\right)_{i \in I}$. Then the following are equivalent:

(1) The random variables $\left(a_{i}\right)_{i \in I}$ are free with respect to $E$.

(2) We have the vanishing of mixed operator-valued free cumulants: For all $n \geq 2$, all $i(1), \ldots, i(n) \in I$, and all $b_{1}, \ldots, b_{n-1} \in \mathcal{B}$ we have

$$
\kappa_{n}^{E}\left(a_{i(1)} b_{1}, \ldots, a_{i(n-1)} b_{n-1}, a_{i(n)}\right)=0
$$

whenever there are $1 \leq k, l \leq n$ such that $i(k) \neq i(l)$.

If we transfer this characterization from the $\kappa_{n}^{E}$ to $\kappa_{\pi}^{E}$ then freeness of the $a_{i}$ implies that $\kappa_{\pi}^{E}\left[a_{i(1)}, \ldots, a_{i(n)}\right]$ can only be non-zero when all the $i$-indices belonging to the same block are equal. It will be convenient to have a notation at hand which encodes that information.

Notation 2.11. For $n \in \mathbb{N}$ and an $n$-tuple $\mathbf{i}=(i(1), \ldots, i(n))$ we denote by ker $\mathbf{i} \in \mathcal{P}(n)$ that partition of $1, \ldots, n$ which is determined by

$$
k \text { and } l \text { are in the same block } \quad \Leftrightarrow \quad i(k)=i(l) .
$$

With this notation we have: if $\left(a_{i}\right)_{i \in I}$ are free with respect to $E$, then $\kappa_{\pi}^{E}\left[a_{i(1)}, \ldots, a_{i(n)}\right]$ can only be non-zero for $\operatorname{ker} \mathbf{i} \geq \pi$. Note that ker $\mathbf{i}$ is in general a possibly crossing partition.

\section{Operator-VAlued FREe RANDOM VARIABles are invariant under Quantum Permutations}

We will now first prove the "easy" direction of our de Finetti theorem, namely that random variables which are free with respect to a conditional expectation $E$ are invariant under quantum permutations with respect to any $\varphi$ which is compatible with $E$. In contrast to the other direction this can be done in a purely algebraic frame, thus we will treat this implication in the context of an arbitrary 
non-commutative probability space. Note also that this implication does actually not require that our sequence is infinite. This will only be crucial for the other implication.

Proposition 3.1. Let $(\mathcal{A}, \varphi)$ be a noncommutative probability space, $\mathcal{B} \subset \mathcal{A}$ a unital subalgebra, and $E: \mathcal{A} \rightarrow \mathcal{B}$ a conditional expectation such that $\varphi=\varphi \circ E$. Consider a sequence $\left(x_{i}\right)_{i \in \mathbb{N}}$ in $\mathcal{A}$ which is identically distributed and free with respect to $E$. Then the joint distribution of the sequence $\left(x_{i}\right)_{i \in \mathbb{N}}$ with respect to $\varphi$ is invariant under quantum permutations.

Proof. Fix $n, k$ and $\mathbf{i}=(i(1), \ldots, i(n))$ with $1 \leq i(1), \ldots, i(n) \leq k$. We have

$$
\begin{aligned}
& \sum_{j(1), \ldots, j(n)=1}^{k} u_{i(1) j(1)} \cdots u_{i(n) j(n)} \cdot \varphi\left(x_{j(1)} \cdots x_{j(n)}\right) \\
= & \sum_{j(1), \ldots, j(n)=1}^{k} u_{i(1) j(1)} \cdots u_{i(n) j(n)} \cdot \varphi\left(E\left[x_{j(1)} \cdots x_{j(n)}\right]\right) \\
= & \sum_{j(1), \ldots, j(n)=1}^{k} u_{i(1) j(1)} \cdots u_{i(n) j(n)} \cdot \varphi\left(\sum_{\pi \in N C(n)} \kappa_{\pi}^{E}\left[x_{j(1)}, \ldots, x_{j(n)}\right]\right) \\
= & \sum_{\pi \in N C(n)} \sum_{j(1), \ldots, j(n)=1}^{k} u_{i(1) j(1)} \cdots u_{i(n) j(n)} \cdot \varphi\left(\kappa_{\pi}^{E}\left[x_{j(1)}, \ldots, x_{j(n)}\right]\right) .
\end{aligned}
$$

Now we note that because of the vanishing of mixed cumulants for free variables the term $\kappa_{\pi}^{E}\left[x_{j(1)}, \ldots, x_{j(n)}\right]$ is only non-vanishing if ker $\mathbf{j} \geq \pi$, where $\mathbf{j}=(j(1), \ldots, j(n))$. Furthermore, by the identical distribution with respect to $E$ of our random variables, for any $\mathbf{j}$ with $\operatorname{ker} \mathbf{j} \geq \pi$ the term $\kappa_{\pi}^{E}\left[x_{j(1)}, \ldots, x_{j(n)}\right]$ has the same value, which we denote by $\kappa_{\pi}^{E}$. Thus we can continue the above calculation as follows:

$$
\begin{aligned}
\sum_{j(1), \ldots, j(n)=1}^{k} u_{i(1) j(1)} \cdots & u_{i(n) j(n)} \cdot \varphi\left(x_{j(1)} \cdots x_{j(n)}\right) \\
= & \sum_{\pi \in N C(n)} \varphi\left(\kappa_{\pi}^{E}\right) \sum_{\substack{j(1), \ldots, j(n)=1, \ldots, k \\
\operatorname{ker} \mathbf{j} \geq \pi}} u_{i(1) j(1)} \cdots u_{i(n) j(n)} .
\end{aligned}
$$

The sum over $j(1), \ldots, j(n)$ with ker $\mathbf{j} \geq \pi$ means that we sum for each block of $\pi$ independently over one $j$-variable. Since $\pi$ is non-crossing at least one of its blocks is an interval, i.e., of the form $\{p, p+1, p+2, \ldots, p+s\}$ for some $1 \leq p \leq p+s \leq k$. Then we have $j(p)=j(p+1)=\cdots=j(p+s)$, the sum over this variable is independent of the other sums; and it only involves

$$
\sum_{j=1}^{k} u_{i(p) j} u_{i(p+1) j} \cdots u_{i(p+s) j} .
$$

Because of the orthogonality of different elements in the same row of $u=\left(u_{i j}\right)_{i, j=1}^{k}$, the term $u_{i(p) j} u_{i(p+1) j} \cdots u_{i(p+s) j}$ is zero for any $j$ unless $i(p)=i(p+1)=\cdots=$ $i(p+s)$. In the latter case, $u_{i(p) j} u_{i(p+1) j} \cdots u_{i(p+s) j}=u_{i(p) j}$ and the sum over $j$ just gives 1 . In this way we are left with the same problem as before but with the 
positions $p, p+1, \ldots, p+s$ removed. For $\pi$ we have just removed one of its interval blocks. Since $\pi$ is non-crossing, we can now find another interval block in the new partition and repeat the above argument. In this way we can do all the summations over the blocks of $\pi$ in an inductive way. In each step the $i$-indices must agree on the considered block of $\pi$ to get a non-vanishing contribution. If they do then the summation over the $j$-index for this block gives 1 . So we get in the end that

$$
\sum_{\substack{j(1), \ldots, j(n)=1, \ldots, k \\
\operatorname{ker} \mathbf{j} \geq \pi}} u_{i(1) j(1)} \cdots u_{i(n) j(n)}=\left\{\begin{array}{ll}
1, & \text { ker } \mathbf{i} \geq \pi \\
0, & \text { otherwise }
\end{array} .\right.
$$

Thus, by recalling that $\kappa_{\pi}^{E}$ is equal to $\kappa_{\pi}^{E}\left[x_{i(1)}, \ldots, x_{i(n)}\right]$ for any $i$ with $\operatorname{ker} \mathbf{i} \geq \pi$, we have

$$
\begin{aligned}
\sum_{j(1), \ldots, j(n)=1}^{k} u_{i(1) j(1)} \cdots u_{i(n) j(n)} & \cdot \varphi\left(x_{j(1)} \cdots x_{j(n)}\right) \\
& =\sum_{\substack{\pi \in N C(n) \\
\operatorname{ker} \mathbf{i} \geq \pi}} \varphi\left(\kappa_{\pi}^{E}\right)=\varphi\left(\sum_{\substack{\pi \in N C(n) \\
\operatorname{ker} \mathbf{i} \geq \pi}} \kappa_{\pi}^{E}\right) \\
& =\varphi\left(\sum_{\substack{\pi \in N C(n) \\
\operatorname{ker} \mathbf{i} \geq \pi}} \kappa_{\pi}^{E}\left[x_{i(1)}, \ldots, x_{i(n)}\right]\right) \\
& =\varphi\left(E\left[x_{i(1)} \cdots x_{i(n)}\right]\right)=\varphi\left(x_{i(1)} \cdots x_{i(n)}\right) .
\end{aligned}
$$

\section{Properties of the Conditional expeCtation ONTO THE TAIL ALGEBRA}

In order to make the step from quantum exchangeability to freeness with amalgamation we need some more analytic structure.

Notation 4.1. Consider a $W$-probability space $(\mathcal{A}, \varphi)$, i.e., $\mathcal{A}$ is a von Neumann algebra and $\varphi$ is a faithful normal state on $\mathcal{A}$. Consider a sequence of random variables $\left(x_{i}\right)_{i \in \mathbb{N}}$ in $\mathcal{A}$.

1) We denote by $\mathcal{A}_{\infty}$ the von Neumann subalgebra generated by $\left(x_{i}\right)_{i \in \mathbb{N}}$, and by $\varphi_{\infty}$ the restriction of $\varphi$ to $\mathcal{A}_{\infty}$.

2) The tail algebra of the sequence $\left(x_{i}\right)_{i \in \mathbb{N}}$ is given by

$$
\mathcal{A}_{\text {tail }}:=\bigcap_{n=1}^{\infty} v N\left(x_{k} \mid k \geq n\right),
$$

where $v N\left(x_{k} \mid k \geq n\right) \subset \mathcal{A}$ is the von Neumann algebra generated by all $x_{k}$ with $k \geq n$.

$\mathcal{A}_{\text {tail }}$ is a von Neumann subalgebra of $\mathcal{A}_{\infty}$ (and thus of $\mathcal{A}$ ). In special cases, $\mathcal{A}_{\text {tail }}$ might be trivial, i.e., equal to $\mathbb{C} 1$, but in general it can be bigger. In any case, if our sequence is exchangeable, then there exists the unique $\varphi_{\infty}$-preserving conditional expectation $E: \mathcal{A}_{\infty} \rightarrow \mathcal{A}_{\text {tail }}$. ( $\varphi_{\infty}$-preserving means of course that $\varphi_{\infty} \circ E=\varphi_{\infty}$.) This is clear if $\varphi$ is a trace. (In this case one does of course not need the exchangeability and one can introduce $E$ directly as a map from $\mathcal{A}$ onto $\mathcal{A}_{\text {tail. }}$.) The general case, which allows non-tracial states, is treated in Koe1 and we adapt a proof from therein for the convenience of the reader. 
Proposition 4.2. Let $(\mathcal{A}, \varphi)$ be a $W^{*}$-probability space and suppose the sequence $\left(x_{i}\right)_{i \in \mathbb{N}} \subset \mathcal{A}$ is exchangeable. Then there exists the $\varphi_{\infty}$-preserving conditional expectation $E$ from $\mathcal{A}_{\infty}$ onto $\mathcal{A}_{\text {tail }}$.

Proof. We can assume that $\mathcal{A}$ is generated by $\left(x_{i}\right)_{i \in \mathbb{N}}$, i.e., that $\mathcal{A}=\mathcal{A}_{\infty}$. Now exchangeability implies the stationarity of $\left(x_{i}\right)_{i \in \mathbb{N}}$ and thus the existence of an endomorphism $\alpha$ of $\mathcal{A}$ such that

$$
\varphi \circ \alpha=\varphi \quad \text { and } \quad \alpha\left(x_{i}\right)=\alpha\left(x_{i+1}\right) .
$$

Let $\mathcal{A}_{I}:=v N\left(x_{i} \mid i \in I\right)$ for $I \subset \mathbb{N}$ and suppose $a, b \in \bigcup_{|I|<\infty} \mathcal{A}_{I}$. Consequently we can assume $a \in \mathcal{A}_{I}$ and $b \in \mathcal{A}_{J}$ such that there exists $N \in \mathbb{N}$ with $I \cap(J+N)=\emptyset$. We infer from exchangeability that $\varphi\left(b \alpha^{n}(a)\right)=\varphi\left(b \alpha^{n+1}(a)\right)$ for all $n \geq N$. Due to minimality this establishes the limit

$$
\lim _{n \rightarrow \infty} \varphi\left(b \alpha^{n}(a)\right)
$$

on the weak*-dense ${ }^{*}$-algebra $\bigcup_{|I|<\infty} \mathcal{A}_{I}$. A standard approximation argument ensures now the existence of this limit for $a, b \in \mathcal{A}$, using the norm density of the functionals $\{\varphi(b \cdot) \mid b \in \mathcal{A}\}$ and the boundedness of the set $\left\{\alpha^{n}(a) \mid n \geq 0\right\}$. We conclude from this that the pointwise limit of the sequence $\left(\alpha^{n}\right)_{n \in \mathbb{N}}$ (in the weak operator topology) defines a linear map $Q: \mathcal{A} \rightarrow \mathcal{A}$ such that $Q(\mathcal{A}) \subset \mathcal{A}_{\text {tail }}$.

It is easily seen that the linear map $Q$ enjoys

$$
\varphi=\varphi \circ Q \quad \text { and } \quad\|Q(a)\| \leq\|a\| \text { for } a \in \mathcal{A} .
$$

Thus $Q$ is a conditional expectation from $\mathcal{A}$ onto $\mathcal{A}_{\text {tail }}$, if we can ensure that $Q(a)=$ $a$ for all $a \in \mathcal{A}_{\text {tail }}$ (see, e.g., [Tak $)$. To this end let $a \in \mathcal{A}_{\text {tail }}$ and $b \in \bigcup_{|I|<\infty} \mathcal{A}_{I}$. We infer from $\mathcal{A}_{\text {tail }} \subset \alpha^{N}(\mathcal{A})$ and $\mathcal{A}_{[N, \infty)} \subset \alpha^{N}(\mathcal{A})$ for all $N \in \mathbb{N}$ that there exists some $N \in \mathbb{N}$ such that $a \in \alpha^{N}(\mathcal{A})$ and $b \in \mathcal{A}_{[0, N-1]}$. We approximate $a \in \mathcal{A}$ in the weak operator topology by a sequence $\left(a_{k}\right)_{k \in \mathbb{N}} \subset \bigcup_{|I|<\infty} \alpha^{N}\left(\mathcal{A}_{I}\right)$ and conclude further from the definition of $Q$ and from exchangeability that

$$
\varphi(b Q(a))=\lim _{k} \varphi\left(b Q\left(a_{k}\right)\right)=\lim _{k} \lim _{n} \varphi\left(b \alpha^{n}\left(a_{k}\right)\right)=\lim _{k} \varphi\left(b a_{k}\right)=\varphi(b a) .
$$

This shows that $Q(a)=a$ for all $a \in \mathcal{A}_{\text {tail }}$. Thus $Q$ is the conditional expectation of $\mathcal{A}$ onto $\mathcal{A}_{\text {tail }}$ with respect to $\varphi$, which we denote from now on by $E$.

Our main goal will be to show that quantum exchangeability implies freeness with respect to this ( $\varphi_{\infty}$-preserving) conditional expectation $E: \mathcal{A}_{\infty} \rightarrow \mathcal{A}_{\text {tail }}$. Note that, in the non-tracial case, we do not define $E$ on $\mathcal{A}$, but only on $\mathcal{A}_{\infty} \subset \mathcal{A}$. This is no problem, however, since all our statements on distribution and freeness with respect to $\mathrm{E}$ involve only elements from $\mathcal{A}_{\infty}$. So, in the present section and in Section 5 the conditional expectation $E$ will always be understood as introduced in Proposition 4.2. If the reader prefers, she may throughout assume that $\mathcal{A}$ is generated by the considered sequence of random variables, i.e., that $\mathcal{A}=\mathcal{A}_{\infty}$ and $\varphi=\varphi_{\infty}$.

Let us first check that quantum exchangeability with respect to $\varphi$ extends to the same property with respect to $E$.

Proposition 4.3. Let $(\mathcal{A}, \varphi)$ be a $W^{*}$-probability space, $\left(x_{i}\right)_{i \in \mathbb{N}}$ a sequence in $\mathcal{A}$, and $E$ the conditional expectation onto the corresponding tail algebra $\mathcal{A}_{\text {tail }}$. Assume that the joint distribution of $\left(x_{i}\right)_{i \in \mathbb{N}}$ with respect to $\varphi$ is invariant under quantum permutations. Then the same is true for the joint distribution of $\left(x_{i}\right)_{i \in \mathbb{N}}$ with respect 
to $E$, i.e., for each $k \in \mathbb{N}$ and $u=\left(u_{i j}\right)_{i, j=1}^{k}$ the generating matrix of $A_{s}(k)$, we have for all $n \in \mathbb{N}$, all $1 \leq i(1), \ldots, i(n) \leq k$ and all $b_{2}, \ldots, b_{n} \in \mathcal{A}_{\text {tail }}$ that

$$
\begin{aligned}
E\left[x_{i(1)} b_{2} x_{i(2)}\right. & \left.\cdots b_{n} x_{i(n)}\right] \\
& =\sum_{j(1), \ldots, j(n)=1}^{k} u_{i(1) j(1)} \cdots u_{i(n) j(n)} \cdot E\left[x_{j(1)} b_{2} x_{j(2)} \cdots b_{n} x_{j(n)}\right] .
\end{aligned}
$$

More generally, for any $p_{1}, \ldots, p_{n} \in \mathcal{A}_{\text {tail }}\langle X\rangle$ we have

$$
\begin{aligned}
& E\left[p_{1}\left(x_{i(1)}\right) p_{2}\left(x_{i(2)}\right) \cdots p_{n}\left(x_{i(n)}\right)\right] \\
& \quad=\sum_{j(1), \ldots, j(n)=1}^{k} u_{i(1) j(1)} \cdots u_{i(n) j(n)} \cdot E\left[p_{1}\left(x_{j(1)}\right) p_{2}\left(x_{j(2)}\right) \cdots p_{n}\left(x_{j(n)}\right)\right] .
\end{aligned}
$$

Proof. Fix $n, k, i(1), \ldots, i(n)$. Because of $\left.\varphi\right|_{\mathcal{A}_{\infty}}=\varphi_{\infty}=\varphi_{\infty} \circ E$, (3) will follow if we can show that

$$
\begin{aligned}
\varphi\left(b_{1} x_{i(1)} b_{2} x_{i(2)} \cdots b_{n} x_{i(n)}\right) \\
=\sum_{j(1), \ldots, j(n)=1}^{k} u_{i(1) j(1)} \cdots u_{i(n) j(n)} \cdot \varphi\left(b_{1} x_{j(1)} b_{2} x_{j(2)} \cdots b_{n} x_{j(n)}\right)
\end{aligned}
$$

for all $b_{1}, \ldots, b_{n} \in \mathcal{A}_{\text {tail }}$. This will follow if we can show Equation (5) for $b_{1}, \ldots, b_{n}$ of the form $x_{r(1)} \cdots x_{r(p)}$ for $p \in \mathbb{N}$ and all $r(1), \ldots, r(p) \geq k+1$. (Note that those $b_{1}, \ldots, b_{n}$ are not from the tail algebra, but we can use them to approximate elements from $\mathcal{A}_{\text {tail }}$. Indeed, by Kaplansky's theorem, these approximation can be done on a norm bounded set, where the multiplication of elements is continuous in the strong operator topology.) Fix such a choice of $b_{1}, \ldots, b_{n}$ and let $N$ be the maximum of all indices appearing in the product $b_{1} x_{i(1)} b_{2} x_{i(2)} \cdots b_{n} x_{i(n)}$ (written as a product in $x$ 's). We extend now the $u$ from $A_{s}(k)$ to a matrix $\tilde{u}=\left(\tilde{u}_{i j}\right)_{i, j=1}^{N}$ according to

$$
\tilde{u}_{i j}=\left\{\begin{array}{ll}
u_{i j}, & \text { if } 1 \leq i, j \leq k \\
\delta_{i j}, & \text { otherwise }
\end{array} .\right.
$$

Then this $\tilde{u}$ satisfies the defining relations of $A_{s}(N)$ and the quantum exchangeability of $x_{1}, \ldots, x_{N}$ under the action of $\tilde{u}$ yields exactly Equation (5). (Note that a priori we also get factors $\tilde{u}_{r j}$ corresponding to the factors $x_{r}$ of the $b$ 's, however, all those will just give a $\delta_{r j}$ contribution, as in this case $r \geq k+1$; thus the $b$ 's from the left side of the equation will just reproduce on the right side of the equation. Also the summation over the $j(m)$-indices for the $x_{i(m)}$ will a priori be from 1 to $N$, but the factor $\tilde{u}_{i(m) j(m)}$ restricts this to the range from 1 to $k$, since $i(m) \leq k$.)

Equation (4) follows from (3) by multilinearity and by checking that we have compatibility of our formulas under multiplying two $x_{i}$ together and under inserting a factor $b \in \mathcal{A}_{\text {tail }}$. But this is clear from the relations of the $u_{i j}$; the first compatibility follows from

$$
\sum_{j(r), j(r+1)=1}^{k} u_{i j(r)} u_{i j(r+1)}=\sum_{j(r)=1}^{k} u_{i j(r)}
$$

and the second one from $\sum_{j=1}^{k} u_{i j}=1$. 
It is clear that the same arguments work also for the case of exchangeability. Since we will use this version in the proof of Proposition 4.5, let us state it here explicitly for later use.

Proposition 4.4. Let $(\mathcal{A}, \varphi)$ be a $W^{*}$-probability space, $\left(x_{i}\right)_{i \in \mathbb{N}}$ a sequence in $\mathcal{A}$, and $E$ the conditional expectation onto the corresponding tail algebra $\mathcal{A}_{\text {tail }}$. Assume that the joint distribution of $\left(x_{i}\right)_{i \in \mathbb{N}}$ with respect to $\varphi$ is invariant under classical permutations. Then the same is true for the joint distribution of $\left(x_{i}\right)_{i \in \mathbb{N}}$ with respect to $E$, i.e., for each $k \in \mathbb{N}$, we have for all $n \in \mathbb{N}$, all $1 \leq i(1), \ldots, i(n) \leq k$ and all $b_{2}, \ldots, b_{n} \in \mathcal{A}_{\text {tail }}$ that

$$
E\left[x_{i(1)} b_{2} x_{i(2)} \cdots b_{n} x_{i(n)}\right]=E\left[x_{\sigma(i(1))} b_{2} x_{\sigma(i(2))} \cdots b_{n} x_{\sigma(i(n))}\right]
$$

for each permutation $\sigma \in S_{k}$.

More generally, for any $p_{1}, \ldots, p_{n} \in \mathcal{A}_{\text {tail }}\langle X\rangle$ we have

$$
\begin{aligned}
& E\left[p_{1}\left(x_{i(1)}\right) p_{2}\left(x_{i(2)}\right) \cdots p_{n}\left(x_{i(n)}\right)\right] \\
&=E\left[p_{1}\left(x_{\sigma(i(1))}\right) p_{2}\left(x_{\sigma(i(2))}\right) \cdots p_{n}\left(x_{\sigma(i(n))}\right)\right]
\end{aligned}
$$

for each permutation $\sigma \in S_{k}$.

In the next section we will show how the quantum exchangeability of $E$ will imply freeness with respect to $E$. For this we will need as an important ingredient the following factorization property of $E$. This is actually a consequence of the classical exchangeability property with respect to $\varphi$ and was shown in Koe1 for more general situations. To establish this desired factorization property it is crucial to work with an infinite sequence of random variables (see also Remark 5.2). In order to make the present paper self-contained we provide the proof for this factorization in our case. For more details and generalizations one should see Koe1. A related finite version of that result was also considered in Lemma 2.6 of $[\mathrm{AL}$.

Proposition 4.5. Let $(\mathcal{A}, \varphi)$ be a $W^{*}$-probability space and $\left(x_{i}\right)_{i \in \mathbb{N}}$ a sequence in $\mathcal{A}$ whose joint distribution is invariant under classical permutations. Let $E$ be the conditional expectation onto the tail algebra $\mathcal{A}_{\text {tail }}$ of the sequence (see Proposition 4.2). Then $E$ has the following factorization property: for all $n \in \mathbb{N}$, all polynomials $p_{1}, \ldots, p_{n} \in \mathcal{A}_{\text {tail }}\langle X\rangle$ and all $i(1), \ldots, i(n) \in \mathbb{N}$ we have

$$
\begin{aligned}
& E\left[p_{1}\left(x_{i(1)}\right) \cdots p_{l}\left(x_{i(l)}\right) \cdots p_{n}\left(x_{i(n)}\right)\right] \\
& \quad=E\left[p_{1}\left(x_{i(1)}\right) \cdots E\left[p_{l}\left(x_{i(l)}\right)\right] \cdots p_{n}\left(x_{i(n)}\right)\right]
\end{aligned}
$$

whenever $i(l)$ is different from all the other $i(r)(r \neq l)$.

Note that exchangeability with respect to $\varphi$ does not imply a factorization property for $\varphi$, but only for the conditional expectation $E$. This is of course responsible for the fact that we get freeness with respect to $E$ and not with respect to $\varphi$ in our noncommutative de Finetti theorem.

Proof. Without restriction we will assume in the following that $\mathcal{A}$ is generated by our sequence, i.e., $\mathcal{A}=\mathcal{A}_{\infty}=v N\left(x_{i} \mid i \in \mathbb{N}\right)$. By $L^{2}(\mathcal{A}, \varphi)$ we will denote the GNS Hilbert space corresponding to $\varphi$, equipped with the inner product $\langle a, b\rangle=\varphi\left(a^{*} b\right)$.

The exchangeability of our sequence implies then that we can define on $L^{2}(\mathcal{A}, \varphi)$ an isometric shift $\alpha$ given by

$$
\alpha\left(x_{i(1)} \cdots x_{i(n)}\right)=x_{i(1)+1} \cdots x_{i(n)+1} .
$$


Restricted to $\mathcal{A} \subset L^{2}(\mathcal{A}, \varphi)$, this shift maps $\mathcal{A}$ into itself and acts there as an endomorphism. Let us denote the fixed point algebra of this shift by

$$
\mathcal{A}_{\alpha}:=\{a \in \mathcal{A} \mid \alpha(a)=a\} .
$$

Clearly, $\mathcal{A}_{\alpha} \subset \mathcal{A}_{\text {tail }}$. We want to show that also $\mathcal{A}_{\text {tail }} \subset \mathcal{A}_{\alpha}$. For this, fix $b \in \mathcal{A}_{\text {tail }}$ and consider, for $m \in \mathbb{N}$ and $r(1), \ldots, r(m) \in \mathbb{N}$, the moment $\varphi\left(x_{r(1)} \cdots x_{r(m)} b\right)$. By approximating $b$ with noncommutative polynomials in $\left\{x_{k} \mid k>\max (r(1), \ldots, r(m))\right\}$ and using the exchangeability of the $\left(x_{i}\right)_{i \in \mathbb{N}}$, one sees that

$$
\varphi\left(x_{r(1)} \cdots x_{r(m)} b\right)=\varphi\left(x_{r(1)} \cdots x_{r(m)} \alpha(b)\right)
$$

for all $m \in \mathbb{N}, r(1), \ldots, r(m) \in \mathbb{N}$. But then one also has

$$
\varphi(a b)=\varphi(a \alpha(b))
$$

for all $a \in \mathcal{A}$ and hence $b=\alpha(b)$. Since this is true for any $b \in \mathcal{A}_{\text {tail }}$ we actually have that $\mathcal{A}_{\alpha}=\mathcal{A}_{\text {tail }}$. Thus Proposition 4.2 entails that the $\varphi$-preserving conditional expectation $E_{\alpha}$ from $\mathcal{A}$ onto $\mathcal{A}_{\alpha}$ exists and equals the conditional expectation $E$ onto the tail algebra.

We recall next that the mean ergodic theorem of von Neumann implies that we have for all all $a \in \mathcal{A}$

$$
\lim _{m \rightarrow \infty} \frac{1}{m} \sum_{i=1}^{m} \alpha^{i}(a)=E_{\alpha}[a]=E[a],
$$

in the strong operator topology (see, e.g., Koe1]).

Now let us consider the situation as in our proposition. By Proposition 4.4 we have exchangeability of $E$ according to (6); this means that we have in our situation

$$
E\left[p_{1}\left(x_{i(1)}\right) \cdots p_{l}\left(x_{i(l)}\right) \cdots p_{n}\left(x_{i(n)}\right)\right]=E\left[p_{1}\left(x_{i(1)}\right) \cdots p_{l}\left(x_{i}\right) \cdots p_{n}\left(x_{i(n)}\right)\right]
$$

for any $i>N:=\max \{i(1), \ldots, i(n)\}$. But then we also have

$$
\begin{aligned}
E\left[p_{1}\left(x_{i(1)}\right)\right. & \left.\cdots p_{l}\left(x_{i(l)}\right) \cdots p_{n}\left(x_{i(n)}\right)\right] \\
& =\frac{1}{m} \sum_{i=N+1}^{N+m} E\left[p_{1}\left(x_{i(1)}\right) \cdots p_{l}\left(x_{i}\right) \cdots p_{n}\left(x_{i(n)}\right)\right] \\
& =E\left[p_{1}\left(x_{i(1)}\right) \cdots\left(\frac{1}{m} \sum_{i=N+1}^{N+m} p_{l}\left(x_{i}\right)\right) \cdots p_{n}\left(x_{i(n)}\right)\right] .
\end{aligned}
$$

But

$$
\frac{1}{m} \sum_{i=N+1}^{N+m} p_{l}\left(x_{i}\right)=\frac{1}{m} \sum_{i=1}^{m} \alpha^{i}\left(p_{l}\left(x_{N}\right)\right)
$$

converges by the mean ergodic theorem to $E\left[p_{l}\left(x_{N}\right)\right]=E\left[p_{l}\left(x_{i(l)}\right)\right]$ and thus we get

$$
\begin{array}{r}
E\left[p_{1}\left(x_{i(1)}\right) \cdots p_{l}\left(x_{i(l)}\right) \cdots p_{n}\left(x_{i(n)}\right)\right] \\
=E\left[p_{1}\left(x_{i(1)}\right) \cdots E\left[p_{l}\left(x_{i(l)}\right)\right] \cdots p_{n}\left(x_{i(n)}\right)\right]
\end{array}
$$

Note again for the convergence argument that multiplication on norm bounded sets is continuous in the strong operator topology. 


\section{INVARIANCE UNDER QUANTUM PERMUTATIONS IMPLIES FREENESS OVER THE TAIL ALGEBRA}

We will now provide the proof of the implication $(\mathrm{a}) \Longrightarrow(\mathrm{b})$ of Theorem 1.1 Throughout this section $E$ will denote the conditional expectation as introduced in Proposition 4.2

Let us first address the identical distribution of the $x_{i}$ with respect to E. For this we actually need only the classical exchangeability.

Proposition 5.1. Let $(\mathcal{A}, \varphi)$ be a $W^{*}$-probability space and consider a sequence $\left(x_{i}\right)_{i \in \mathbb{N}}$ in $\mathcal{A}$. Assume that the joint distribution of $\left(x_{i}\right)_{i \in \mathbb{N}}$ with respect to $\varphi$ is invariant under classical permutations. Then the sequence $\left(x_{i}\right)_{i \in \mathbb{N}}$ is identically distributed with respect to the conditional expectation $E$ onto the tail algebra of $\left(x_{i}\right)_{i \in \mathbb{N}}$.

Proof. This is just a special case of Proposition 4.4.

Now we will address the freeness property. For this one needs, as in the classical case, an infinite sequence of random variables. One should, however, note that the only way in which this infinity enters is via the factorization property of Proposition 4.5 (which relied in the end on the mean ergodic theorem). If this factorization property is assumed then it is feasible that a more elaborated version of the following arguments is also applicable to finite sequences of random variables.

We will check that $x_{1}, x_{2}, \ldots$ are free with respect to $E$ by verifying the defining relations for freeness. So let us consider $n \in \mathbb{N}$ and polynomials $p_{1}, \ldots, p_{n} \in$ $\mathcal{A}_{\text {tail }}\langle X\rangle$ such that $E\left[p_{i}\left(x_{1}\right)\right]=0$ for all $i=1, \ldots, n$. Then we have to show that for all $i(1) \neq i(2) \neq \cdots \neq i(n)$

$$
E\left[p_{1}\left(x_{i(1)}\right) \cdots p_{n}\left(x_{i(n)}\right)\right]=0 .
$$

We will prove this (for fixed $n$ and $p_{1}, \ldots, p_{n}$ ) by induction over the number of blocks of ker i, starting from the biggest number and going down in steps of one. To get started consider the biggest number of blocks, which is $n$. Then all $i(1), \ldots, i(n)$ are different and, by an iterated application of the factorization property, Proposition 4.5, we have

$$
E\left[p_{1}\left(x_{i(1)}\right) \cdots p_{n}\left(x_{i(n)}\right)\right]=E\left[p_{1}\left(x_{i(1)}\right)\right] \cdots E\left[p_{n}\left(x_{i(n)}\right)\right]=0 .
$$

Now assume, for some $r$, we have proved that $E\left[p_{1}\left(x_{i(1)}\right) \cdots p_{n}\left(x_{i(n)}\right)\right]=0$ whenever $i(1) \neq i(2) \neq \cdots \neq i(n)$ and ker $\mathbf{i}$ has at least $r+1$ blocks. We want to show the same for the case that ker $\mathbf{i}$ has $r$ blocks.

By Proposition 4.3, we have

$$
\begin{aligned}
& E\left[p_{1}\left(x_{i(1)}\right) \cdots p_{n}\left(x_{i(n)}\right)\right] \\
& =\sum_{j(1), \ldots, j(n)=1}^{k} u_{i(1) j(1)} \cdots u_{i(n) j(n)} \cdot E\left[p_{1}\left(x_{j(1)}\right) \cdots p_{n}\left(x_{j(n)}\right)\right] \\
& =\sum_{\pi \in \mathcal{P}(n)} \sum_{\substack{j(1), \ldots, j(n)=1 \\
\operatorname{ker} \mathbf{j}=\pi}}^{k} u_{i(1) j(1)} \cdots u_{i(n) j(n)} \cdot E\left[p_{1}\left(x_{j(1)}\right) \cdots p_{n}\left(x_{j(n)}\right)\right]
\end{aligned}
$$

Let us first observe that j's where two neighboring indices are the same do not contribute; this follows from the fact that $u_{i(s) j(s)} u_{i(s+1) j(s+1)}=0$ if $j(s)=j(s+1)$ 
because $i(s) \neq i(s+1)$. Thus we only have to sum over $\mathbf{j}=(j(1), \ldots, j(n)$ in the above sum for which $j(1) \neq j(2) \neq \cdots \neq j(n)$. But for those our induction hypothesis applies and thus we see that in the summation over $\pi \in \mathcal{P}(n)$ we can restrict to $\pi$ which have at most $r$ blocks. Since $E\left[p_{1}\left(x_{i(1)}\right) \cdots p_{n}\left(x_{i(n)}\right)\right]$ is invariant under permutations, we can fix specific (different) $i$-values for the $r$ blocks of ker $\mathbf{i}$; let us take $1,3,5, \ldots, 2 r-1$ for them.

Let us now choose $k=2 r$ and a specific $u=\left(u_{i j}\right)_{i, j=1}^{2 r}$, namely

$$
u=\left(\begin{array}{ccccccc}
q_{1} & 1-q_{1} & 0 & 0 & \ldots & 0 & 0 \\
1-q_{1} & q_{1} & 0 & 0 & \ldots & 0 & 0 \\
0 & 0 & q_{2} & 1-q_{2} & \ldots & 0 & 0 \\
0 & 0 & 1-q_{2} & q_{2} & \ldots & 0 & 0 \\
\vdots & \vdots & \vdots & \vdots & \ddots & \vdots & \vdots \\
0 & 0 & 0 & 0 & \ldots & q_{r} & 1-q_{r} \\
0 & 0 & 0 & 0 & \ldots & 1-q_{r} & q_{r}
\end{array}\right)
$$

where $q_{1}, \ldots, q_{r}$ are arbitrary projections. With this choice of $u$ we have that for a non-vanishing $u_{i j}$ the $j$-value determines the $i$-value (since we only have the odd numbers as possible $i$-values), i.e., we have $\operatorname{ker} \mathbf{j} \leq \operatorname{ker} \mathbf{i}$; thus in the sum (8) we can restrict to $\pi \leq$ ker i. But since we also restricted to $\pi$ with at most $r$ blocks, we are just left with the one possibility $\pi=\operatorname{ker} \mathbf{i}$, i.e., with the above $u$ we can continue (8) as follows:

$$
\begin{aligned}
E\left[p_{1}\left(x_{i(1)}\right)\right. & \left.\cdots p_{n}\left(x_{i(n)}\right)\right] \\
& =\sum_{\substack{j(1), \ldots, j(n)=1 \\
\operatorname{ker} \mathbf{j}=\operatorname{ker} \mathbf{i}}}^{2 r} u_{i(1) j(1)} \cdots u_{i(n) j(n)} \cdot E\left[p_{1}\left(x_{j(1)}\right) \cdots p_{n}\left(x_{j(n)}\right)\right] \\
& =\left(\sum_{\substack{j(1), \ldots, j(n)=1 \\
\operatorname{ker} \mathbf{j}=\operatorname{ker} \mathbf{i}}}^{2 r} u_{i(1) j(1)} \cdots u_{i(n) j(n)}\right) \cdot E\left[p_{1}\left(x_{i(1)}\right) \cdots p_{n}\left(x_{i(n)}\right)\right]
\end{aligned}
$$

In the last step we have used the fact that, because of the identical distribution of the $x_{i}$ 's with respect to $E$, the term $E\left[p_{1}\left(x_{j(1)}\right) \cdots p_{n}\left(x_{j(n)}\right)\right]$ depends only on ker $\mathbf{j}$.

If we can show that

$$
\sum_{\substack{j(1), \ldots, j(n)=1 \\ \text { ker } \mathbf{j}=\operatorname{ker} \mathbf{i}}}^{2 r} u_{i(1) j(1)} \cdots u_{i(n) j(n)}
$$

is different from 1 , then this implies that $E\left[p_{1}\left(x_{i(1)}\right) \cdots p_{n}\left(x_{i(n)}\right)\right]$ has to vanish, and we are done.

Note that if ker $\mathbf{i}$ is non-crossing then the sum (10) is actually equal to 1 for any $u$ satisfying the relations of the quantum permutation group. However, if ker $\mathbf{i}$ is non-crossing then the condition $i(1) \neq i(2) \neq \cdots \neq i(n)$ implies that ker $\mathbf{i}$ must have at least one singleton, i.e., one i-index appears only once and then the factorization property (77) gives right away that $E\left[p_{1}\left(x_{i(1)}\right) \cdots p_{n}\left(x_{i(n)}\right)\right]=0$. Thus we can restrict to crossing ker $\mathbf{i}$ when considering (10).

Note also that if all the $u_{i j}$ in (10) commute, then we will actually get 1 (independent of whether ker $\mathbf{i}$ is crossing or non-crossing); this shows that invariance under 
usual permutations is (clearly) not strong enough to imply freeness. We have to invoke some real quantum permutations, i.e., we should choose the $q_{1}, \ldots, q_{r}$ in (9) as non-commuting. However, it suffices to take just two of them as non-commuting. Since ker $\mathbf{i}$ is crossing we can choose two blocks which have a crossing. For those two blocks we choose some non-commuting projections $p$ and $q$, whereas for all the other blocks we choose their projections as 1 . Then the sum (10) reduces to

$$
(p q)^{s}+(p(1-q))^{s}+((1-p) q)^{s}+((1-p)(1-q))^{s}
$$

or to

$$
(p q)^{s} p+(p(1-q))^{s} p+((1-p) q)^{s}(1-p)+((1-p)(1-q))^{s}(1-p)
$$

for some $s \geq 2$. It is clear that this is not equal to 1 for generic projections $p$ and $q$. Actually it is fairly easy to see that these expressions are equal to 1 if and only if $p$ and $q$ commute.

This finishes the proof of the implication $[(\mathrm{a}) \Longrightarrow(\mathrm{b})$ in Theorem 1.1

Remark 5.2. As already mentioned before, our noncommutative de Finetti theorem is not true for a finite number of random variables. To infer freeness from quantum exchangeability one needs, as in the classical case, infinitely many variables. To prove that claim we will in the following present an example, which can be considered as an analogue to classical urn models without replacement.

Consider the quantum permutation group $A_{s}(n)$ itself, with defining matrix $u=$ $\left(u_{i j}\right)_{i, j=1}^{n}$. Then, by a fundamental result of Woronowicz [Wor, there exists a normalized Haar functional $\psi: A_{s}(n) \rightarrow \mathbb{C}$. Consider now the $G N S$ representation of $A_{s}(n)$ with respect to $\psi$; this gives a $W^{*}$-probability space $(\mathcal{A}, \psi)$, where $\mathcal{A}$ is the weak closure of $A_{s}(n)$. The defining invariance property of the Haar functional implies that each column of $u=\left(u_{i j}\right)_{i j=1}^{n}$ is invariant under quantum permutations from $A_{s}(n)$. To be concrete, let us consider the first column, $u_{11}, \ldots, u_{n 1}$. These $n$ elements are quantum exchangeable with respect to $\psi$. However, we claim that there does not exist a conditional expectation $E$ from $\mathcal{A}$ onto a von Neumann subalgebra $\mathcal{B} \subset \mathcal{A}$ with $\psi \circ E=\psi$, such that $u_{11}, \ldots, u_{n 1}$ are identically distributed and free with respect to $E$. Assume the contrary. Since $u_{11} u_{21}=0$, we would have

$$
0=E\left[u_{11} u_{21}\right]=E\left[u_{11}\right] E\left[u_{21}\right]=E\left[u_{11}\right] E\left[u_{11}\right] .
$$

Since $E\left[u_{11}\right]$ is selfadjoint this implies that $E\left[u_{11}\right]=0$, and thus also

$$
\psi\left(u_{11}\right)=\psi\left(E\left[u_{11}\right]\right)=0 .
$$

However, $u_{11}=u_{11} u_{11}^{*}$ and $\psi$ is faithful, thus $u_{11}=0$, which is a contradiction.

\section{ACKNOWLEDGEMENT}

We thank Franz Lehner for some helpful comments on an earlier version of the manuscript.

\section{REFERENCES}

[AL] L. Accardi, Y. G. Lu: A continuous version of de Finetti's theorem. Ann. Probab. 21 (1993), 1478-1493.

[BC] T. Banica, B. Collins: Integration over quantum permutation groups. J. Funct. Anal. 242 (2007), 641-657.

[BBC] T. Banica, J. Bichon, B. Collins: Quantum permutation groups: a survey. Banach Center Publ. 78 (2007), 13-34. 
[Leh] F. Lehner: Cumulants in noncommutative probability theory. IV. De Finetti's Theorem, $L^{p}$-inequalities. J. Funct. Anal. 239 (2006), 214-246.

[HP] F. Hiai, D. Petz: The Semicircle Law, Free Random Variables and Entropy. Math. Surveys and Monogr. 77, AMS 2000

[Kal] O. Kallenberg: Probabilistic Symmetries and Invariance Principles. Probability and Its Applications. Springer-Verlag, 2005.

[Koe1] C. Köstler: A noncommutative extended de Finetti theorem. Preprint. (electronic) arXiv:0806.3621v1 [math.0A], 2008.

[Koe2] C. Köstler: On Lehner's 'free' noncommutative analogue of de Finetti's theorem. Preprint. (electronic) arXiv:0806.3632v1 [math.0A], 2008.

[NS] A. Nica, R. Speicher: Lectures on the Combinatorics of Free Probability. London Mathematical Society Lecture Note Series, no. 335. Cambridge University Press, 2006.

[Spe] R. Speicher: Combinatorial theory of the free product with amalgamation and operatorvalued free probability theory. Mem. Amer. Math. Soc., vol. 132, no. 627, pp. x+88, 1998.

[Tak] M. Takesaki: Theory of Opertor Algebras II. Encyclopaedia of Mathematical Sciences. Springer, 2003.

[Voi] D. Voiculescu: Operations on certain non-commutative operator-valued random variables. Astérisque, no. 232, pp. 243-275, 1995.

[VDN] D. Voiculescu, K. Dykema, A. Nica: Free Random Variables. AMS, Providence, Rhode Island, 1992.

[Wan] S. Wang: Quantum symmetry groups of finite spaces. Comm. Math. Phys. 195 (1998), 195-211.

[Wor] S.L. Woronowicz: Compact matrix pseudogroups. Comm. Math. Phys. 111 (1987), 613665.

University of Illinois at Urbana-Champaign, Department of Mathematics, Altgeld Hall, 1409 West Green Street, Urbana, 61801, USA

E-mail address: koestler@uiuc.edu

Queen's University, Department of Mathematics and Statistics, Jeffery Hall, Kingston, ON K7L 3N6, CANADA

E-mail address: speicher@mast.queensu.ca 